\title{
La tombe monumentale de Grotte Scalina et la Tuscia viterbaise
}

Campagne de fouilles 2018

Vincent Jolivet et Edwige Lovergne

\author{
(2) OpenEdition \\ Journals \\ Édition électronique \\ URL : http://journals.openedition.org/cefr/2801 \\ DOI : 10.4000/cefr.2801 \\ ISSN : 2282-5703 \\ Éditeur \\ École française de Rome
}

Référence électronique

Vincent Jolivet et Edwige Lovergne, «La tombe monumentale de Grotte Scalina et la Tuscia viterbaise », Chronique des activités archéologiques de l'École française de Rome [En ligne], Italie centrale, mis en ligne le 09 mai 2019, consulté le 01 août 2019. URL : http://journals.openedition.org/cefr/2801 ; DOI 10.4000/cefr.2801

Ce document a été généré automatiquement le 1 août 2019.

(c) École française de Rome 


\title{
La tombe monumentale de Grotte Scalina et la Tuscia viterbaise
}

\author{
Campagne de fouilles 2018 \\ Vincent Jolivet et Edwige Lovergne
}

\section{NOTE DE L'AUTEUR}

Ont participé aux travaux de la campagne 2018 : Fanny Auffret (Paris), Anne-Lise Baylé (université de Paris I), Alice Baud (université de Paris I), Hélène Bédoire (Université de Tours), Hugo Cador (université de Paris I), Marjolaine Bénaich (ENS, Paris), Thomas Broise (Aix-en-Provence), Julien Bruyère (cellule archéologique du Conseil départemental des Ardennes), Anaïs Daumont-Marx (Paris), Joël Defrance-Jublot (Lyon), Manuel de Souza (université de Saint-Étienne), Benjamin Houal (Paris), Dominique Maisonneuve (Paris), Marlène Nazarian-Trochet (université de Paris Nanterre), Johan Pinvidic (Aix-en-

Provence), Alexandre Rabot (HiSoMa, Lyon).

1 Au terme de la septième campagne de fouilles sur le site de la tombe monumentale de Grotte Scalina, nous nous sommes consacrés à la recherche de l'emplacement de l'hypogée originaire de cette tombe, à l'approfondissement du parallèle offert par la tombe Lattanzi de Norchia, et à différentes opérations de fouilles ponctuelles, programmées (Civita Musarna), d'urgence (Tuscania, tombe des Cochons Noirs) ou préventives (Tuscania, loc. Le Mandrie), ainsi qu'à des travaux de documentation (Castel d'Asso).

Les travaux se sont déroulés du 2 juillet au 4 août 2018 sous la direction scientifique de Vincent Jolivet et sous la direction opérationnelle d'Edwige Lovergne, en étroite collaboration avec la Soprintendenza Archeologia, Belle Arti e Paesaggio per l'area metropolitana di Roma, la provincia di Viterbo e l'Etruria meridionale (inspectrice: Dott.ssa Maria Letizia Arancio), sous le régime de la collaboration opérative. Les relevés ont été effectués par Filippo Bozzo (Università della Tuscia, Viterbo), ainsi que par Guilhem Chapelin (Centre Jean Bérard, Naples) et François Fouriaux (École française de 
Rome), les vues par drone par Benjamin Houal (Paris). Les recherches géophysiques ont été réalisées d'abord par Stefano Urbini (Istituto Nazionale di Geofisica e Vulcanologia), puis par Gianluca Catanzariti (3DGEOIMAGING). Les travaux de chantier ont été effectués par l'entreprise Enzo Nicolamme de Monterazzano.

Le financement a été assuré par l'UMR 8546 (AOrOc) du CNRS, le Département des Sciences de l'Antiquité de l'École normale supérieure de Paris, l'ANR CAECINA, le Labex TransferS (programme Investissements d'avenir ANR-10-IDEX-000102 PSL* et ANR10LABX-0099). Maria et Enzo Pepponi, à Grotte Scalina, et Lorenzo Caponetti, à Tuscania, ont généreusement contribué à la réalisation de ces différentes opérations.

\section{Prospections géophysiques : Grotte Scalina}

4 Les différents indices invitant à penser que les deux hypogées fouillés les années précédentes n'ont jamais été utilisés au cours des deux premières générations d'utilisation de la tombe, probablement entre 320 et 280 av. J.-C. (cf. notre précédente (hronique), du fait de la mauvaise qualité du sol naturel rencontré par les aménageurs au niveau de celles-ci, nous ont amenés à émettre l'hypothèse que ce premier hypogée aurait été creusé dans la couche de tuf compact correspondant à la façade du monument ; si tel était bien le cas, son accès devait être un dromos creusé à partir de l'extrémité du plateau qui surmonte la tombe, probablement creusé dans l'axe central du monument.

5 La prospection géophysique réalisée par G. Catanzariti a révélé à cet emplacement la présence d'une anomalie circulaire de $40 \mathrm{~m}$ de diamètre (fig. 1), qui ne semble pouvoir s'expliquer par aucun phénomène de nature géologique (tout ce secteur ayant été bonifié dans les années 1970, la couche de terre labourée repose directement sur le banc de tuf dérasé). L'hypothèse d'une motte médiévale semble peu probable : ce type de site fortifié n'est pas attesté dans le Latium, et il est normalement bien antérieur aux XII ${ }^{\mathrm{e}}$ XIII ${ }^{\mathrm{e}}$ siècles, au cours desquels ce secteur a effectivement été occupé, et défendu par une enceinte et un fossé, bien visible sur la carte de prospection. 
Fig. 1 - Grotte Scalina, prospections géophysiques, carte de synthèse de la zone située au-dessus de la tombe monumentale.

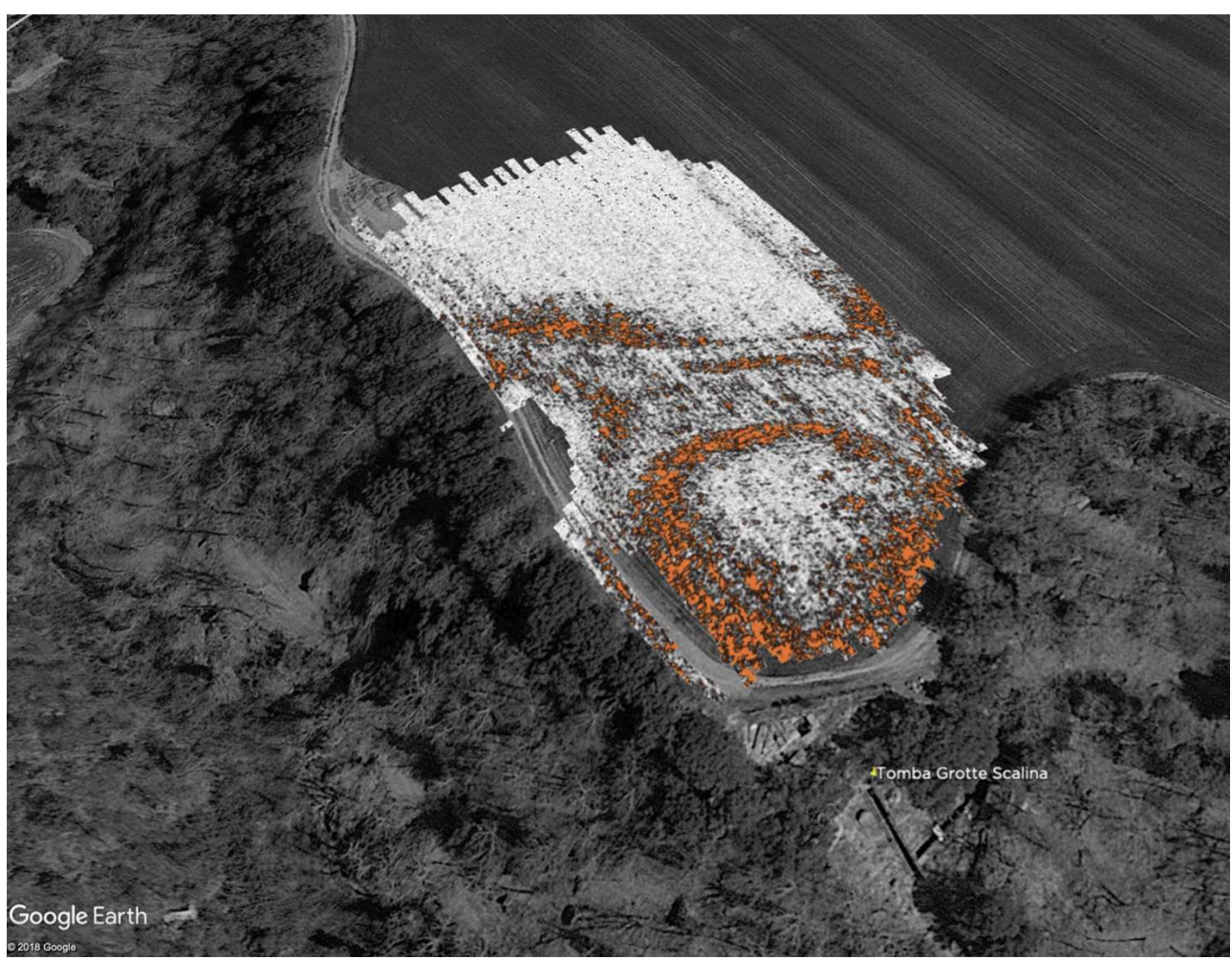

G. Catanzariti.

6 Il ne semble donc pas trop aventureux d'interpréter cette structure comme un tumulus, probablement en liaison directe avec la tombe rupestre dont il aurait protégé l'accès à l'hypogée originaire, tout en signalant la tombe depuis la voie qui reliait Tuscania à Sorrina/Viterbe. Cette création néo-orientalisante ne serait pas isolée : selon un dispositif par ailleurs plus classique, la tombe Torlonia de la nécropole de Monte Abatone, à Cerveteri, appartenant elle aussi à une famille de la très haute aristocratie étrusque, était également surmontée par un tumulus (fig. 2). 
Fig. 2 - Hypothèse interprétative de la structure circulaire de la carte géophysique de synthèse comme un tumulus, et comparaison avec la tombe Torlonia de Cerveteri, contemporaine.
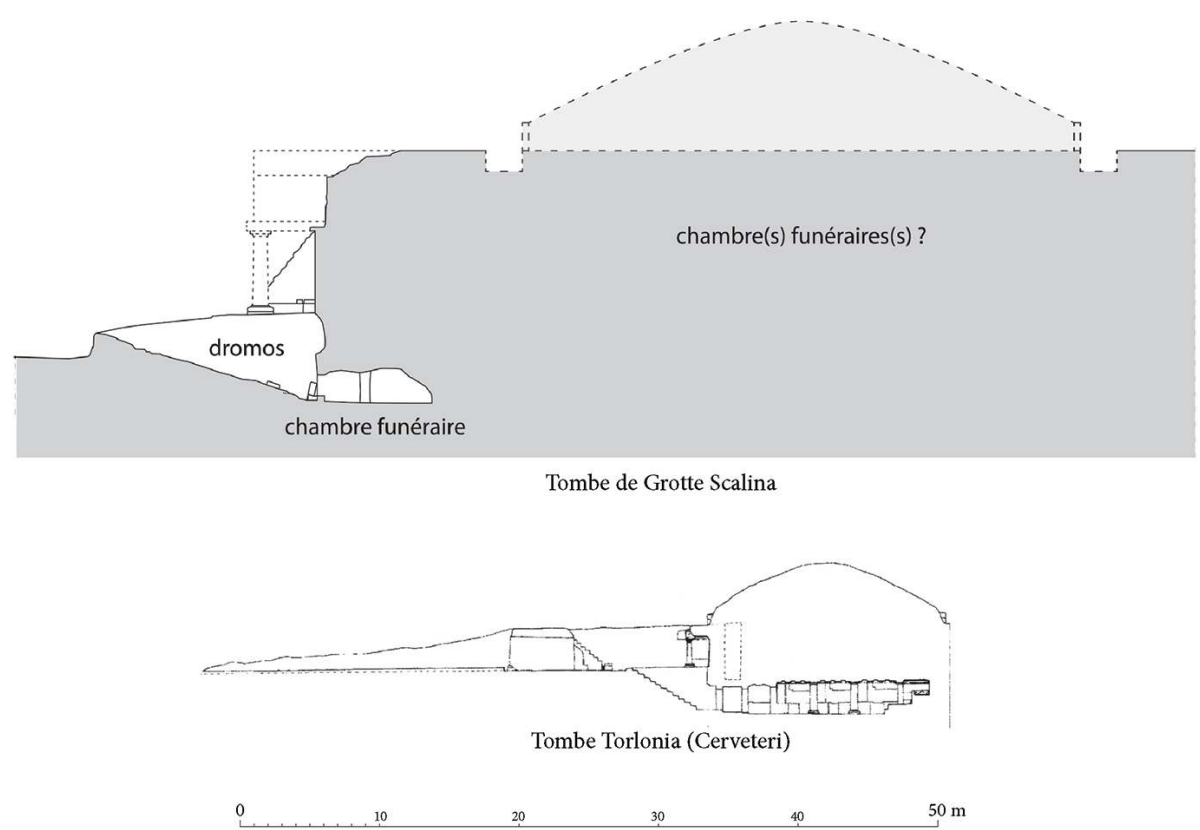

G. Chapelin.

\section{Opérations programmées}

\section{Norchia, tombe Lattanzi}

7 Certains des problèmes posés par la restitution de la tombe de Grotte Scalina pourraient être résolus en reprenant l'étude de la tombe jumelle de Norchia, fouillée au milieu du XIX siècle, même si leur architecture n'était manifestement pas semblable en tout point. En effet, tandis qu'à Grotte Scalina la transformation du site en lieu de culte chrétien nous a privés de tous les éléments de destruction du monument - fragments d'architecture, décors, sculptures...-, à Norchia, ceux-ci, qui devraient être encore probablement sur place après l'effondrement de la structure, sont aujourd'hui enfouis au pied du monument.

Le nettoyage réalisé cette année sur la tombe a confirmé l'hypothèse de la présence d'une salle de banquet - mais celle-ci ne comportait que quatre lits (fig. 3), contre six à Grotte Scalina. À gauche de la salle, la sculpture qui garde la tombe doit probablement être interprétée non pas comme un animal réel ou mythique (lion, sphinx, chimère, griffon...), puisque sa patte arrière se termine en sabot, mais comme un taureau, sur lequel des traces jusqu'ici inexpliquées invitent à reconnaitre une figure humaine, enveloppée dans des voiles (fig. 4): il s'agit très probablement d'une représentation de l'enlèvement d'Europe par Zeus (Evre, en étrusque, figure sur un miroir contemporain de Tarquinia), dont la présence en contexte funéraire pourrait s'expliquer par le fait que deux des juges des Enfers, Minos et Rhadamante, naquirent de leur union - le troisième, Éaque, était fils de Zeus et d'Égine. Du côté opposé de la salle de banquet, un grand fragment d'extrémité 
d'aile en nenfro témoigne de la présence d'une statue, probablement de sphinx, symétrique du groupe d'Europe.

Fig. 3 - Norchia, tombe Lattanzi, les deux lits de droite de la salle de banquet.

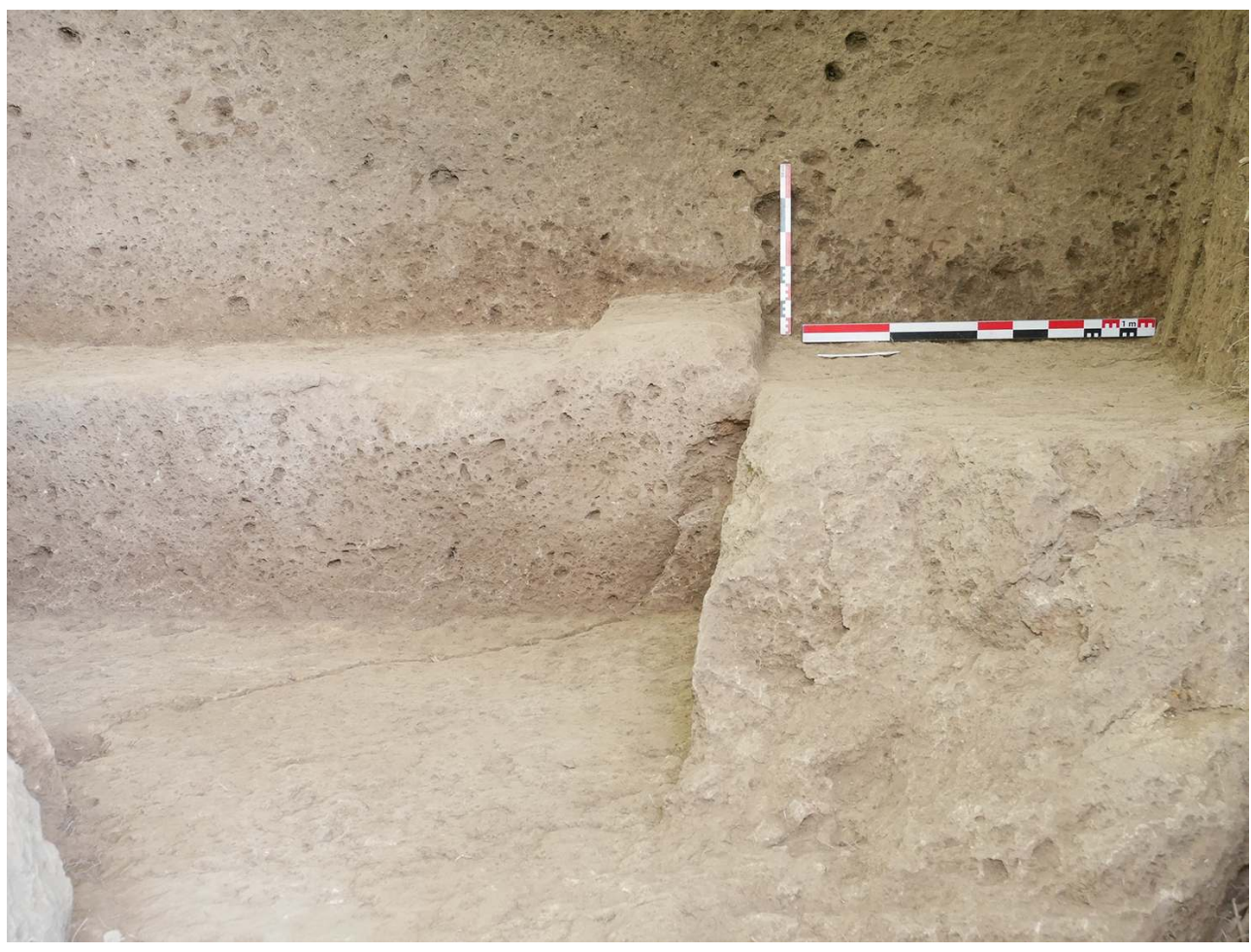

Archives de fouilles 2018 
Fig. 4 - Norchia, tombe Lattanzi, à gauche de la salle de banquet, le rapt d'Europe ?

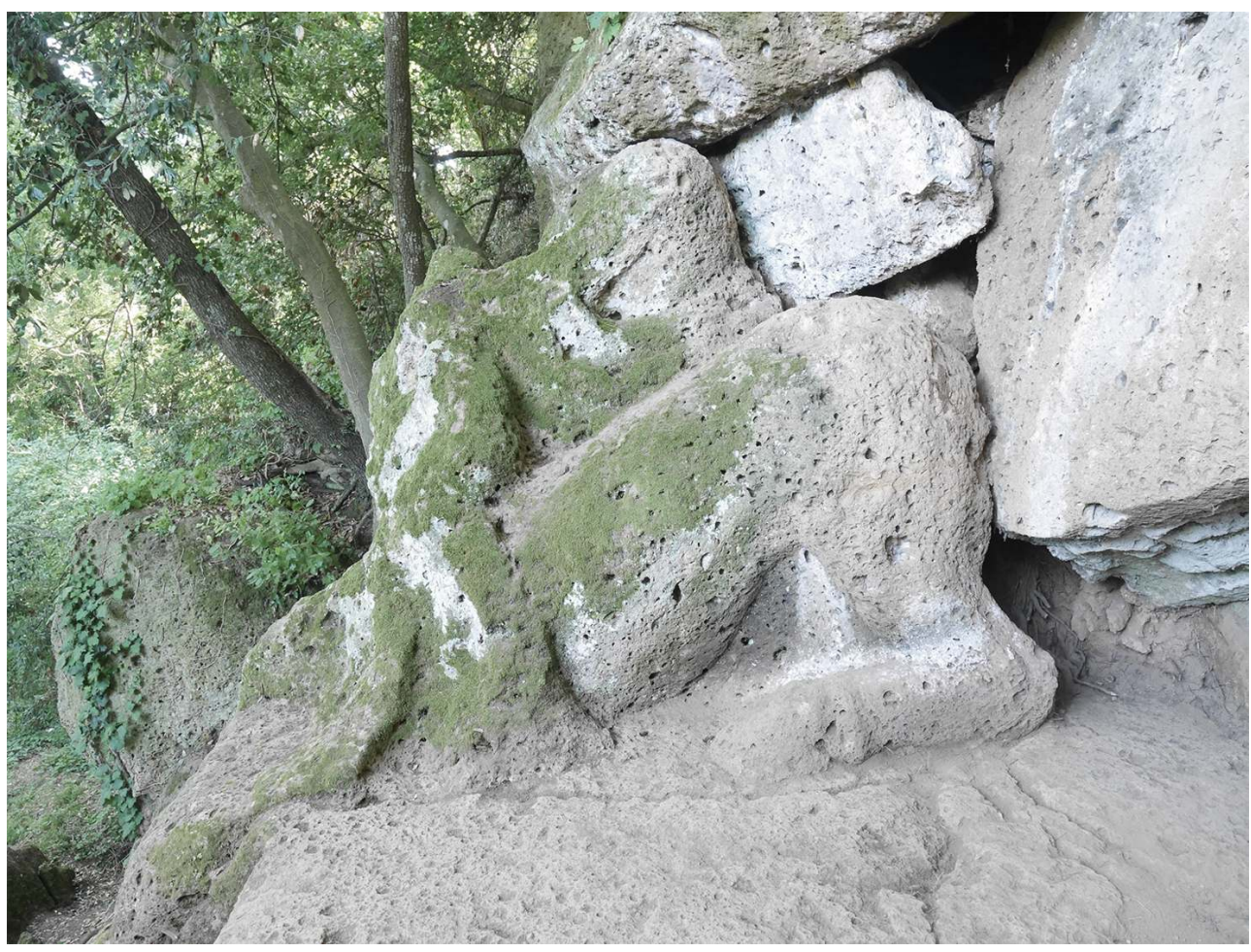

Archives de fouilles 2018

9 La vérification des restitutions proposées pour cette tombe devrait impliquer trois opérations principales : le dégagement de son toit, qui n'était probablement pas plat, mais à double pente; la fouille du secteur situé en contrebas de la tombe, qui devrait livrer tout un ensemble d'informations sur l'architecture et le décor du monument ; un nouveau nettoyage des deux chambres funéraires de l'hypogée, qui permettrait certainement de préciser la datation de la tombe et sa durée d'occupation, pour l'heure entièrement fondée sur les sarcophages qui en ont été extraits.

\section{Musarna, le locus inaugurationis?}

10 Les recherches menées par Giuseppina Cinque (Università degli Studi di Tor Vergata, Dipartimento di Ingegneria) avaient permis d'émettre une hypothèse de localisation du point de départ de l'urbanisation de Musarna (fig. 5), désigné dans la littérature archéologique comme mundus ou locus inaugurationis, et qui pouvait être marqué par des vestiges de sacrifice, une cavité rituelle ou encore, comme à Marzabotto, par un gros galet portant un decussis. 
Fig. 5 - Musarna, hypothèse de localisation du locus inaugurationis.

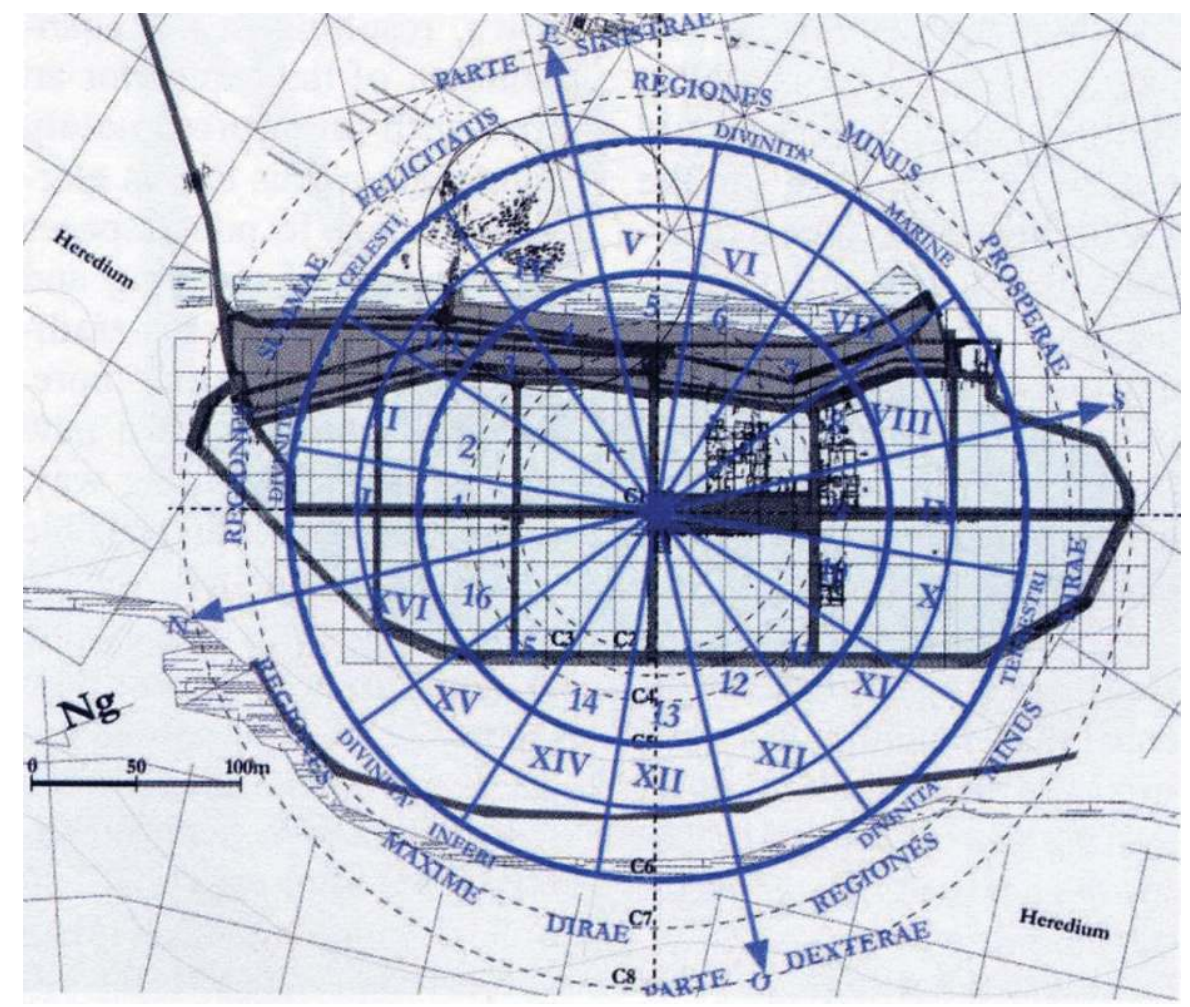

G. Cinque.

Le sondage de $2 \mathrm{~m}$ de côté réalisé à cet emplacement précis, au nord de la place centrale, a seulement permis de mettre au jour dans le banc de tuf, avec un décalage vers le sud-est d'une cinquantaine de centimètres par rapport au point exact estimé (fig. 6), un trou de poteau de $29 \mathrm{~cm}$ de diamètre calé par des pierres et des fragments de tuile, contenant des fragments de charbon de bois, et scellé par un niveau d'époque impériale (fig. 7). Il pourrait donc s'agir du lieu où était fiché le gnomon utilisé au départ des opérations de planification urbaine, pour déterminer les principaux axes de la nouvelle fondation. 
Fig. 6 - Musarna, emplacement du sondage ouvert au nord de la place centrale.
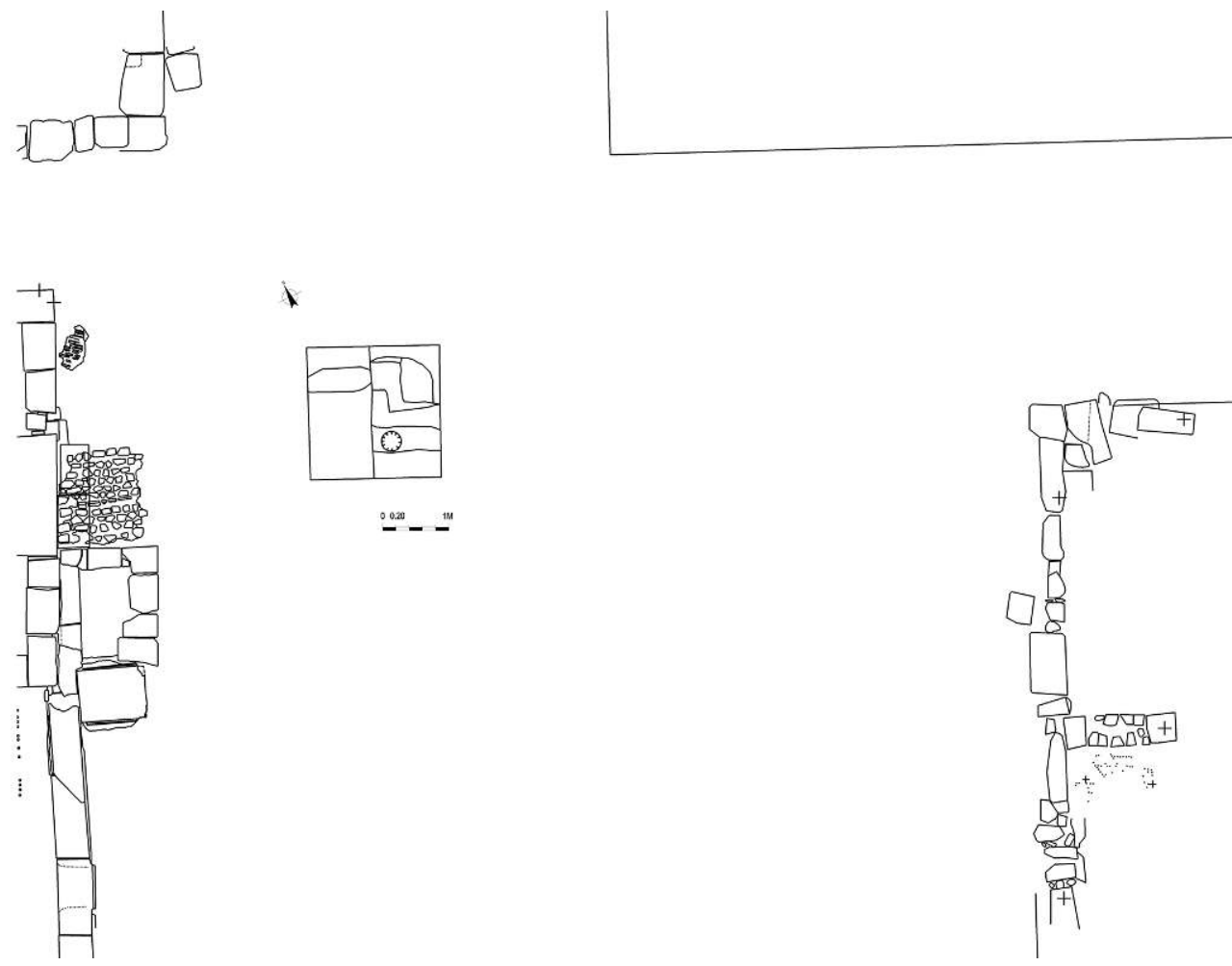

\.

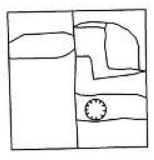

$\stackrel{-1+3}{-1+}$

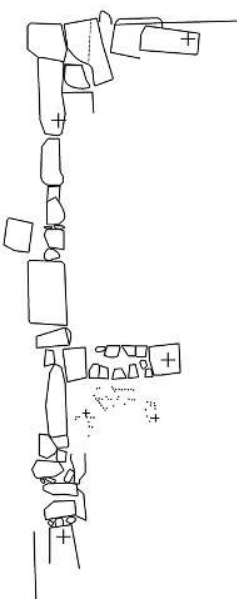

F. Bozzo.

Fig. 7 - Musarna, le trou de poteau du gnomon?

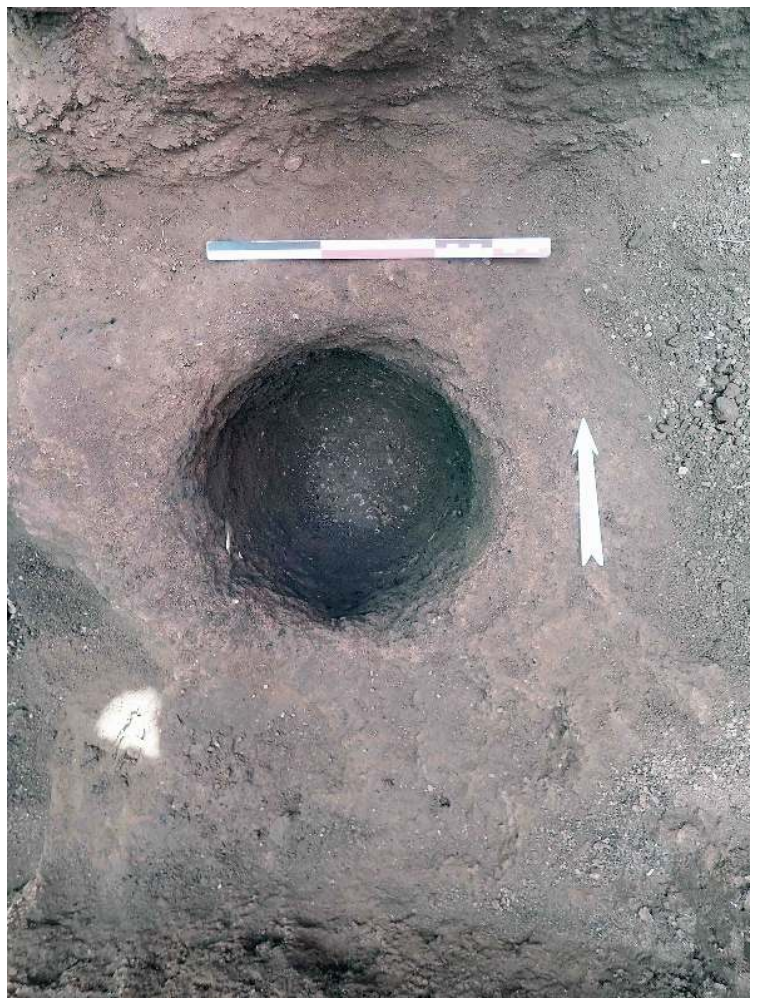

Archives de fouilles 2018

Chronique des activités archéologiques de l'École française de Rome , Italie centrale 
Deux nouveaux sondages ouverts à l'emplacement des deux carrefours opposés, au nord et au sud, pourraient permettre de confirmer cette hypothèse.

\section{Castel d'Asso, les tombes a cassone}

Dans le cadre de l'étude de la typologie particulière des tombes a cassone signalées en dehors de Musarna, nous avons procédé au nettoyage de deux tombes de ce type au sudouest de la nécropole de Castel d'Asso (tombes 3 et 4), de manière à pouvoir en dresser un plan précis qui n'existait pas, faute de fouille de leur comblement.

\section{Opérations d'urgence et préventives}

\section{Tuscania, la tombe des Cochons Noirs}

Au cours de l'hiver 2017-2018, dans le domaine Caponetti, au sud-est de Tuscania, loc. Quarticciolo, un groupe de cochons noirs, élevés en liberté, a mis au jour fortuitement un segment de mur dont il était probable qu'il appartenait à une tombe de la nécropole toute proche de Sasso Pizzuto, hypothèse dont il fallait s'assurer rapidement pour éviter l'intervention sur le site de fouilleurs clandestins.

La fouille a confirmé cette hypothèse, et permis de dégager la partie antérieure d'une tombe construite $a$ dado dont la façade, percée par une porte basse, fait face à la vallée du Marta (fig. 8). À l'extérieur de la tombe, de nombreux tessons (bucchero gris, céramique attique à figures noires) indiquent qu'il s'agit vraisemblablement d'une tombe archaïque. À droite de la façade, une fosse contenait encore une amphore attique à figures noires (face A, Apollon et deux femmes ; face B, départ de guerrier) en position verticale, fermée par une coupe de céramique vernie en noir retournée, et contenant des cendres (fig. 9); la fouille du contenu du vase a été réalisée par Henri Duday, qui a pu établir que les restes de la crémation, très diversement incinérés, avaient été préalablement rassemblés dans un sac en matériau périssable, lui-même déposé dans l'amphore.

Fig. 8 - Tuscania, tombe des Cochons Noirs, photogrammétrie de la façade de la tombe en fin de fouille.

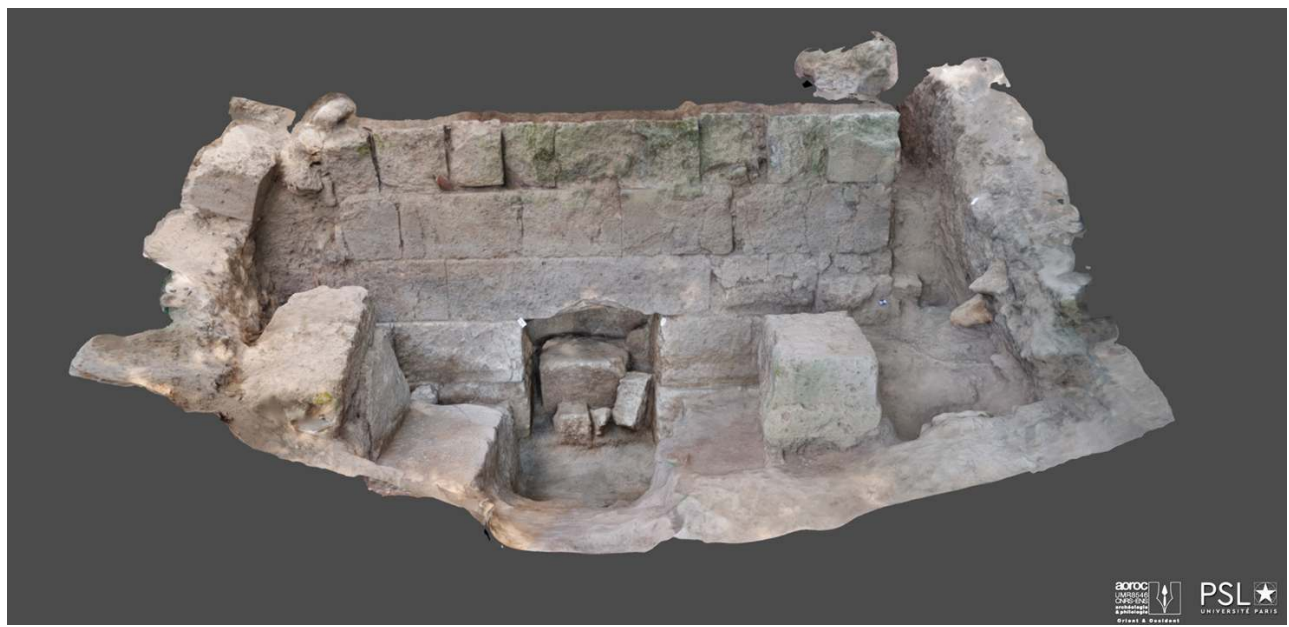

B. Houal. 
Fig. 9 - Tuscania, tombe des Cochons Noirs, la façade de la tombe en cours de dégagement, avec l'amphore attique à figures noires in situ.

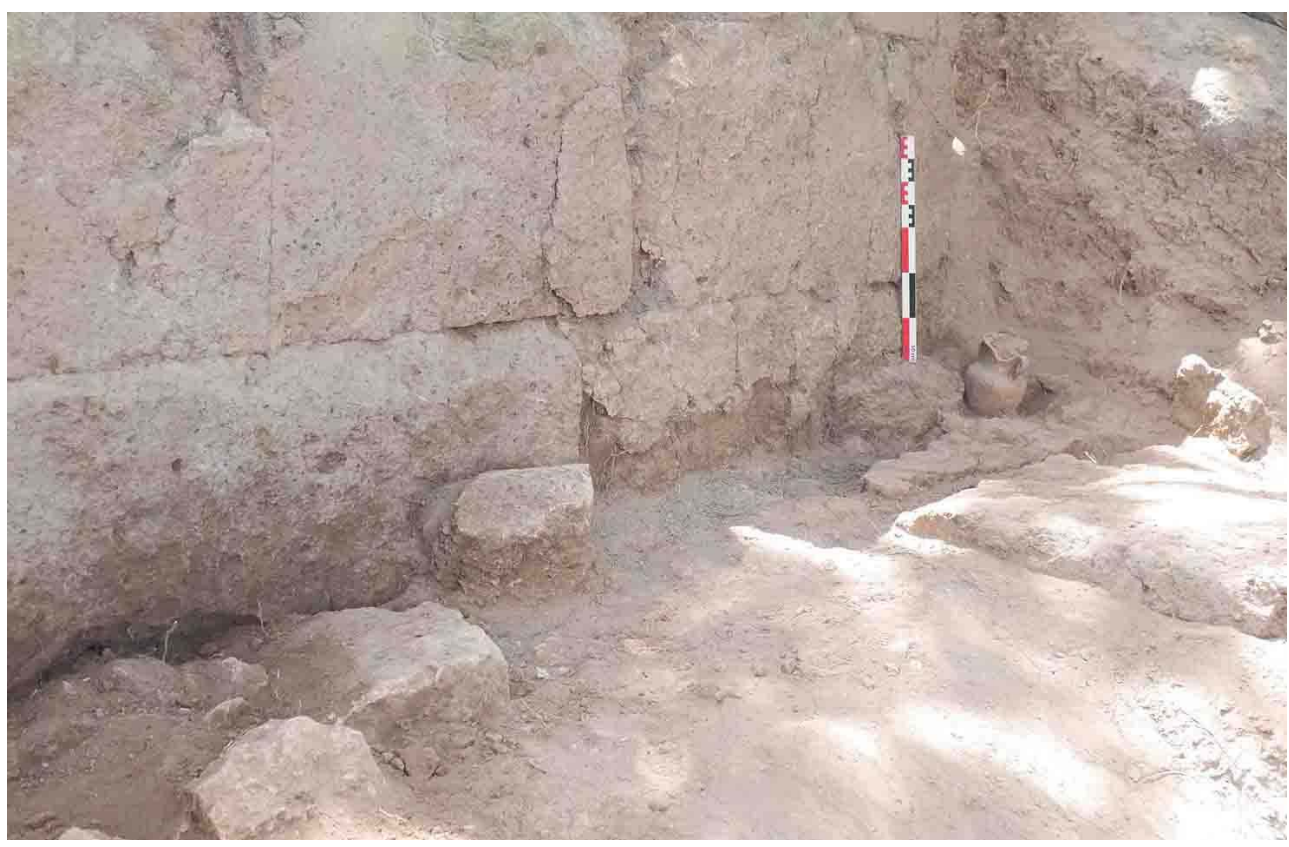

Archives de fouilles 2018.

16 La typologie de la tombe, tout à fait nouvelle pour le site, pourrait documenter ultérieurement les relations étroites qui unissaient Tuscania et Volsinies à l'époque archaïque, déjà bien attestées par le répertoire céramique du site.

L'effondrement de la voûte de la partie antérieure de l'hypogée, derrière la façade, qui pourrait avoir protégé une partie du mobilier de la tombe, n'a pas permis d'en terminer la fouille cette année : elle sera reprise en 2019.

\section{Tuscania, loc. Le Mandrie}

Un projet de carrière a motivé une intervention préventive, réalisée sous forme de tranchées parallèles, sur une extension de huit hectares. Aucun vestige archéologique n'a été mis au jour au cours de cette opération.

\section{BIBLIOGRAPHIE}

V. Jolivet, L. Motta, Musarna: Macchia del Conte Vecchia, REE, dans SE 75, 2009, p. 252-253.

V. Jolivet, E. Lovergne, La tomba rupestre monumentale di Grotte Scalina (VT), dans L. Mercuri,

R. Zaccagnini (dir.), Atti del convegno Etruria in Progress. La ricerca archeologica in Etruria meridionale, Rome, 2014, p. 165-170. 
V. Jolivet, E. Lovergne, Architecture palatiale macédonienne, architecture funéraire étrusque : la tombe monumentale de Grotte Scalina (Viterbe), dans Actes du colloque de Merida, Madrid, 2015, p. 47-50.

V. Jolivet, E. Lovergne, La tombe rupestre monumentale de Grotte Scalina (Étrurie méridionale), dans Revue Archéologique, 61-1, 2016, p. 151-162.

V. Jolivet, E. Lovergne, Etruscan puzzle: the monumental tomb of Grotte Scalina, dans World Archaeology, 80, 2016, p. 26-30.

V. Jolivet, Macedonia and Etruria at the beginning of the Hellenistic period: a direct link, dans

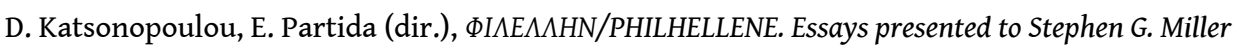

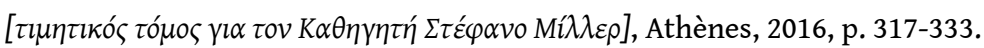

V. Jolivet, E. Lovergne, The monumental tomb of Grotte Scalina: solved and unsolved questions, dans Etruscan News, 18, 2017, p. 10.

C. Berrendonner, G. van Heems, Ager Tarquiniensis (Grotte Scalina), REE, dans StEtr, 80, 2018, sous presse.

G. Amicucci, P. Catalano, V. Jolivet, E. Lovergne, Réinvestissement et pillage d'une tombe monumentale étrusque : Grotte Scalina (Viterbe), dans $9^{e}$ Rencontre du Gaaf, sous presse.

V. Jolivet, Le banquet funéraire dans l'Étrurie rupestre hellénistique, dans L'Etruria delle necropoli rupestri, XXIX convegno di Studi Etruschi ed Italici, sous presse.

V. Jolivet, E. Lovergne, Architecture funéraire étrusque, affirmation gentilice, dans M. Arizza (dir.), Società e pratiche funerarie a Veio, Rome, sous presse.

\section{INDEX}

Mots-clés : Étrurie, Grotte Scalina, époque hellénistique, Norchia, Musarna, Tuscania, Casel d'Asso, tombe rupestre, époque archaïque institutions Soprintendenza Archeologia, Belle Arti e Paesaggio per l'area metropolitana di Roma, Provincia di Viterbo e l'Etruria meridionale, AOrOc (UMR 8546 CNRS), École normale supérieure (Paris)

\section{AUTEURS}

VINCENT JOLIVET

CNRS, AOROC (UMR 8546 CNRS-ENS) - vincent.jolivet@ens.fr

\section{EDWIGE LOVERGNE}

CNRS, AOROC (UMR 8546 CNRS-ENS) - edwigelovergne@hotmail.com 